\title{
Diacronie
}

Studi di Storia Contemporanea

$\mathrm{N}^{\circ} 28,4 \mid 2016$

La voce del silenzio

\section{Emma Fattorini (a cura di), Diplomazia senza eserciti. Le relazioni internazionali della Chiesa di Pio XI}

Donato Di Sanzo

\section{Q OpenEdition \\ 1 Journals}

\section{Edizione digitale}

URL: http://journals.openedition.org/diacronie/4651

DOI: 10.4000/diacronie.4651

ISSN: 2038-0925

Editore

Association culturelle Diacronie

Notizia bibliografica digitale

Donato Di Sanzo, « Emma Fattorini (a cura di), Diplomazia senza eserciti. Le relazioni internazionali della Chiesa di Pio XI », Diacronie [Online], № 28, 4 | 2016, documento 28, Messo online il 29 décembre 2016 consultato il 24 septembre 2020. URL : http://journals.openedition.org/diacronie/4651 ; DOI : https:// doi.org/10.4000/diacronie.4651 


\section{Diacronie}

N. 28 | 4|2016 La voce del silenzio: intelligence, spionaggio e conflitto nel XX secolo

\section{RECENSIONE:}

\section{Emma FATTORINI (a cura di), Diplomazia} senza eserciti. Le relazioni internazionali della Chiesa di Pio XI, Roma, Carocci, 2o13, 227 pp.

a cura di Donato DI SANZO *

Uscito nel 2013 per i tipi di Carocci, Diplomazia senza eserciti. Le relazioni internazionali della Chiesa di Pio XI, a cura di Emma Fattorini, si inserisce nella ormai florida produzione di studi sulla diplomazia vaticana tra le due guerre mondiali, pubblicati dopo che, nel settembre 2006, le carte relative al periodo del pontificato di Achille Ratti (1922-1939), conservate nell'Archivio Segreto Vaticano, sono state rese disponibili alla consultazione degli studiosi ${ }^{1}$. La scelta del titolo reca una "fortunata" efficacia evocativa nell'individuare il filo conduttore che lega i sei saggi contenuti nel volume: nel corso del pontificato rattiano, la crescita della dimensione internazionale della Santa Sede fu perseguita attraverso uno sforzo di mondializzazione della Chiesa condotto senza un apparato militare, nell'esercizio di un potere che, con l'ausilio della terminologia contemporanea, si potrebbe definire soft.

Di particolare interesse è l'introduzione critica della curatrice, che compie anche un lavoro sinottico nella scelta di definire tre categorie di analisi - il comunismo, l'americanismo e il totalitarismo, corrispondenti agli orientamenti politici con cui ebbe a relazionarsi la diplomazia vaticana tra le due guerre mondiali - per tracciare le linee

\footnotetext{
${ }^{1}$ A titolo esemplificativo, si vedano: CHIRON, Yves, Pio XI. Il papa dei patti lateranensi e dell'opposizione ai totalitarismi, Cinisello Balsamo, San Paolo Edizioni, 2006; FATTORINI, Emma, Pio XI, Hitler e Mussolini. La solitudine di un papa, Torino, Carocci, 2007; CECI, Lucia, Il papa non deve parlare. Chiesa, fascismo e guerra d'Etiopia, Roma-Bari, Laterza, 2010; CECI, Lucia, L’interesse superiore. Il Vaticano e l'Italia di Mussolini, Roma-Bari, Laterza, 2013; KERTZER, David, Il patto col diavolo. Mussolini e papa Pio XI. Le relazioni segrete fra il Vaticano e l'Italia Fascista, Milano, Rizzoli, 2014 [Ed. originale The Pope and Mussolini. The Secret History of Pius XI and the Rise of Fascism in Europe, New York, Random House, 2014].
} 
fondamentali del volume e chiarire che i primi tre saggi ne rappresentano il nucleo centrale.

Il primo saggio, di Filippo Frangioni, dal titolo Unione Sovietica e guerra di Spagna: comunismo e Santa Sede (pp. 19-54), infatti, presenta una ricognizione sull'atteggiamento vaticano nei confronti del comunismo e dell'Unione Sovietica negli anni Venti e Trenta. Molto chiara appare la scelta dell'autore di definire l'oggetto della trattazione nella ricostruzione «di alcuni momenti del processo di definizione e condanna del comunismo, attraverso le interpretazioni vaticane della politica estera di Mosca in rapporto al contesto della guerra civile spagnola» ${ }^{2}$. Il riferimento specifico ai fatti di Spagna è opportunamente preceduto da una accurata trattazione dell'evoluzione della politica vaticana nei confronti del comunismo e della situazione in Russia: da un atteggiamento di «realismo politico» che, nel corso degli anni Venti e attraverso la Pontificia Commissione Pro Russia, operò il tentativo «di trovare alcune modalità di rapporto con il nuovo Stato sovietico»3 nella speranza di ristabilire una presenza cattolica nel mondo ortodosso, si passò a una nuova posizione negli anni Trenta che, sotto l’impulso della rivista gesuita «Lettres de Rome sul l'Athéisme Moderne» e del Segretariato gesuita sull'ateismo moderno, univa il sempre presente rigetto della dottrina comunista a una condanna senza mezzi termini dell'Unione Sovietica e della sua politica internazionale. Rispetto a un quadro simile, l'autore, attraverso le fonti dell'Archivio Segreto Vaticano, della Sacra Congregazione per gli Affari Ecclesiastici Straordinari e dell'Archivum Romanum Societatis Iesu, giunge ad individuare la guerra in Spagna come «punto di incontro» dei diversi processi rappresentativi del nuovo giudizio della Santa Sede nei confronti del comunismo, che sarebbe confluito nell'enciclica Divini Redemptoris del marzo del 1937: il ruolo giocato dai sovietici proprio nella vicenda spagnola testimoniava l'esistenza di una «funzione di destabilizzazione internazionale svolta dall'URSS» ${ }^{4}$, mentre le persecuzioni religiose in atto nella penisola iberica conducevano alla «definizione del comunismo come fenomeno essenzialmente antireligioso»5.

Il secondo saggio in volume, di Giulia D’Alessio, intitolato Stati Uniti, Chiesa cattolica e questione sociale (pp. 55-99), si inserisce nel già ampio panorama di studi

\footnotetext{
2 FRANGIONI, Filippo, Unione Sovietica e Guerra di Spagna: comunismo e Santa Sede, in FATTORINI, Emma (a cura di), Diplomazia senza eserciti. Le relazioni internazionali della Chiesa di Pio XI, Roma, Carocci, 2013, p. 21.

3 Ibidem, p. 24.

4 Ibidem, p. 53.

5 Ibidem, p. 54.
} 
sulle relazioni internazionali tra Santa Sede e Stati Uniti tra le due guerre mondiali ${ }^{6}$, attraverso una considerazione documentata della questione sociale interna al cattolicesimo d'oltreoceano e dell'influenza che l'opera di alcuni eminenti cattolici statunitensi e il rapprochement diplomatico tra Santa Sede e amministrazione americana esercitarono sulla formulazione delle politiche del New Deal roosveltiano. A partire da una ricostruzione del pensiero di John Augustine Ryan, uno dei cattolici statunitensi più attivi nella formulazione di un programma di politiche sociali, e attraverso le fonti dell'Archivio Segreto Vaticano e della Franklin Delano Roosevelt Presidential Library, l'autrice ricostruisce l'appoggio che i cattolici, al netto di particolari eccezioni7, recarono nei confronti della politica di riforma dell'amministrazione democratica degli anni Trenta e, soprattutto, dei suoi effetti sulla regolamentazione dei salari e sull'organizzazione dei lavoratori. Un appoggio che promanava dal recepimento della Quadragesimo Anno del 1931, l'enciclica sociale di Pio XI diffusa nel quarantesimo anniversario della Rerum Novarum di Leone XIII, e che coincise con il riavvicinamento diplomatico tra Santa Sede e Stati Uniti culminato nello storico viaggio oltreoceano del Cardinale Segretario di Stato Eugenio Pacelli.

Il terzo saggio, di Luca Ferracci, dal titolo Il Reichskonkordat $e$ il cattolicesimo in Germania, presenta una documentata ricostruzione delle tappe che condussero alla sottoscrizione di un concordato tra Santa Sede e Germania nel 1933. A partire da una dettagliata disquisizione storiografica sul dibattito in merito al ruolo dei cattolici nelle ultime fasi dell'esperienza repubblicana weimariana ${ }^{8}$, l'autore si serve della

\footnotetext{
${ }^{6}$ A titolo esemplificativo, fra le pubblicazioni più recenti, si vedano CASTAGNA, Luca, Un ponte oltre l'oceano. Assetti politici e strategie diplomatiche tra Stati Uniti e Santa Sede nella prima metà del Novecento (1914-1940), Bologna, Il Mulino, 2011 [ed. originale A bridge across the ocean. The United States and the Holy See between two world wars, Washington D.C., The Catholic University of America Press, 2014]; ROSSI, Cristina, Santa Sede e Stati Uniti d'America tra le due guerre: tentativi di dialogo nelle presidenze Harding e Coolidge, in DE LEONARDIS, Massimo (a cura di), Fede e diplomazia. Le relazioni internazionali della Santa Sede nell'età contemporanea, Milano, Educatt, 2014, pp. 97-116.

7 Si veda il paragrafo dedicato alla campagna anti-roosveltiana che il sacerdote di Detroit Charles E. Coughlin condusse negli anni Trenta attraverso la sua trasmissione radiofonica, cfr. D'ALESSIO, Giulia, Stati Uniti, Chiesa cattolica e questione sociale, in FATTORINI, Emma (a cura di), op. cit., pp. 83-91.

${ }^{8} \mathrm{Si}$ fa riferimento qui al dibattito tra la cosiddetta "storiografia della colpa" «che tendeva a enfatizzare le responsabilità cattoliche nell'ascesa e nel sostegno del nazismo» e coloro che, invece, non imputavano responsabilità dirette alla Chiesa tedesca e alla politica cattolica riguardo all'avvento di Hitler al potere. Nel ricostruire la controversia tra lo storico cattolico Konrad Repgen, rappresentante della "storiografia della colpa", e lo storico evangelico Klaus Scholder, l'autore cita, in particolare, i seguenti lavori: BLASCHKE, Olaf, Geischichtsdeutung und Vergangenheitspolitik. Die Kommission für Zeitgeschichte und das Netzwerk kirchenloyaler Katholizismusforscher 1945-200o, in PITTROF, Thomas, SCHMITZ, Walter (herausgegeben von), Freie Anerkennung übergeschichtlicher Bindungen. Katholische Geschichtswahrnehmung im deutschsprachigen Raum des 20. Jahrhunderts, Freiburg, Rombach Verlag, 2010, pp. 479-521; WOLF, Hubert, «Reichskonkordat für
} 
documentazione conservata nell'Archivio Segreto Vaticano e, in particolare, dei fogli d'udienza del Cardinale Segretario di Stato Eugenio Pacelli, per giungere alla conclusione che la Chiesa tedesca e la Santa Sede non ebbero un ruolo diretto nella fine della democrazia in Germania e che non è possibile rintracciare sufficienti evidenze documentarie di un avvenuto accordo tra Hitler e il Vaticano, in virtù del quale il partito cattolico del Centro avrebbe votato a favore della concessione dei pieni poteri al Fürher in cambio della sottoscrizione del concordato del 1933. Molto più equilibrata risulta la posizione di Ferracci, che, al termine del saggio individua fra le ragioni dimostrabili del Reichskonkordat «l'aver pensato di trovare nel radicale contrapporsi del nazismo al materialismo marxista e allo spirito liberale del tempo una ragione per riconoscere in esso elementi di un possibile incontro e di una collaborazione»9.

Esaurito, con i primi tre contributi, il nucleo centrale della trattazione, il volume presenta due saggi di carattere più "compilativo". Il primo, di Magali Della Sudda, reca il titolo Santa Sede e associazionismo cattolico femminile francese e ricostruisce l'atteggiamento della Chiesa transalpina e del Vaticano nei confronti dell'animato mondo delle associazioni cattoliche femminili in Francia nel clima del pontificato di Pio XI, promotore di una regolamentazione e di un sostegno nei confronti dell'Azione Cattolica nei contesti nazionali. Il saggio di Mara Dissegna, intitolato L'apertura delle nunziature ungherese e romena, presenta in maniera abbastanza schematica e fedele alla lettera delle evidenze documentarie citate la vicenda dell'istituzione di un presidio diplomatico della Santa Sede nei due peculiari contesti dell'est europeo.

A chiusura del volume è riportato l'interessante saggio di Marie Levant dal titolo La "mano tesa", La Chiesa di Pio XI e il Fronte popolare, che ricostruisce, sempre con l'ausilio delle fonti dell'Archivio Segreto Vaticano, l'apertura di Pio XI verso i comunisti francesi, la quale, nel clima del Fronte Popolare internazionale di metà anni Trenta e in seguito all'appello alla collaborazione con i cattolici lanciato dal segretario del Partito comunista transalpino Maurice Thorez, fu concepita come il tentativo di non lasciare «ai comunisti il monopolio dell'afflizione per le sofferenze umane» ${ }^{10}$ e non come una generale riconsiderazione dell'atteggiamento vaticano nei confronti del materialismo ateo e delle dottrine socialiste. Originale e appropriata risulta la conclusione dell'autrice, che individua nell'apertura rattiana di fronte alla "mano tesa" dei

Ermächtigungsgesetz? Zur Historisierung der Scholder-Repgen-Kontroverse über das Verhältnis des Vatikans zun Nationalsozialismus», in Vierteljahrshefte für Zeitgeschichte, 6o, 2/2012, pp. 160-200. Cfr. FERRACCI, Luca, Il Reichskonkordat $e$ il cattolicesimo in Germania, in FATTORINI, Emma (a cura di), op. cit., pp. 102-107.

9 Ibidem, p. 133.

${ }^{10}$ LEVANT, Marie, La "mano tesa", la Chiesa di Pio XI e il Fronte Popolare, in FATTORINI, Emma (a cura di), op. cit., p. 192. 
comunisti francesi un «alleggerimento nei confronti, non tanto del comunismo, quanto dei suoi sostenitori» ${ }^{11}$, un atteggiamento che procedette di pari passo con l'inasprimento del giudizio di Pio XI nei confronti dei totalitarismi fascista e nazista.

Diplomazie senza eserciti, a cura di Emma Fattorini, rappresenta un contributo importante per la comprensione della politica internazionale della Santa Sede tra le due guerre mondiali, che, dall'apertura degli archivi vaticani, si è arricchita e continua ad arricchirsi di nuovi e originali lavori. Sembra di scorgere, nelle pagine del volume, almeno nel suo nucleo centrale e nell'originalità di alcuni spunti conclusivi, il tentativo di superare il limite alle ricerche storiche sul pontificato di Pio XI che la curatrice ha individuato, in alcuni casi, nel non aver affrontato la ricognizione sui documenti dell'Archivio Segreto Vaticano «con il respiro dei tempi lunghi, della ricerca sistematica “vecchio stile" ${ }^{12}$.

${ }^{11}$ Ibidem, p. 200.

${ }^{12}$ FATTORINI, Emma (a cura di), op. cit., p. 11. 


\section{* L'autore}

Donato Di Sanzo, attualmente borsista presso l'Istituto Italiano per gli Studi Storici di Napoli, è dottore di ricerca e cultore della materia in Storia Contemporanea presso l'Università degli studi di Salerno. Ha pubblicato Oltre le barricate. Storia, politica, religione e l'Ulster della pace (Roma, Aracne, 2016) e sta lavorando alla pubblicazione di una monografia sulle relazioni diplomatiche tra Santa Sede e Irlanda nel periodo tra le due guerre mondiali.

URL: < http://www.studistorici.com/progett/autori/\#DiSanzo >

\section{Per citare questo articolo:}

DI SANZO, Donato, «Recensione: Emma FATTORINI (a cura di), Diplomazia senza eserciti. Le relazioni internazionali della Chiesa di Pio XI, Roma, Carocci, 2013, 227 pp.», Diacronie. Studi di Storia Contemporanea : La voce del silenzio: intelligence, spionaggio e conflitto nel XX secolo, 29/12/2016,

URL:<http://www.studistorici.com/2016/12/29/sanzo_numero_28/ >

Diacronie Studi di Storia Contemporanea ô www.diacronie.it

Risorsa digitale indipendente a carattere storiografico. Uscita trimestrale.

redazione.diacronie@hotmail.it

Comitato di redazione: Jacopo Bassi - Luca Bufarale - Antonio César Moreno Cantano - Deborah Paci - Fausto Pietrancosta - Alessandro Salvador - Matteo Tomasoni - Luca Zuccolo

Diritti: gli articoli di Diacronie. Studi di Storia Contemporanea sono pubblicati sotto licenza Creative Commons 3.0. Possono essere riprodotti e modificati a patto di indicare eventuali modifiche dei contenuti, di riconoscere la paternità dell'opera e di condividerla allo stesso modo. La citazione di estratti è comunque sempre autorizzata, nei limiti previsti dalla legge. 